\title{
Correlation between Von Mises Strain and Material Thinning in a Hydroformed Sample of Ti35A Aerospace Grade Titanium
}

\author{
Colin Bell ${ }^{1, \text { a) }}$, Ellen Jump ${ }^{2, \text { b) }}$, William Kerr², c), Jonathan Corney ${ }^{1, \text { d), }}$ \\ Nicola Zuelli ${ }^{2, \text { e) }}$, David Savings ${ }^{3, \text { f) }}$ \\ ${ }^{1}$ Design, Manufacture and Engineering Management, University of Strathclyde, 75 Montrose Street, Glasgow G1 \\ $1 \mathrm{XJ}$ \\ ${ }^{2}$ Advanced Forming Research Centre, 85 Inchinnan Drive, Inchinnan, Renfrewshire PA4 9LJ \\ ${ }^{3}$ Rolls-Royce plc, PO Box 31, Derby DE24 8BJ \\ a)Colin.Bell@strath.ac.uk \\ b)Ellen.Jump@ @strath.ac.uk \\ c)William.Kerr@strath.ac.uk \\ d)Jonathan.Corney@strath.ac.uk

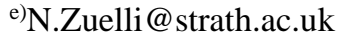

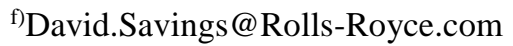

\begin{abstract}
This paper presents the results of an experimental investigation of the strain measured on a sample of Ti35A (commercially pure) titanium that was formed past the point of failure in a hydroforming operation. The sample was etched prior to forming to allow for a strain map of the exterior to be created and examined by using a circle grid analysis (CGA) technique. The sample was scanned post forming with precise optical inspection to ascertain an accurate model of its geometry. This paper discusses the results of the analyses including the full geometric and surface strain measurements. This paper then compares material thinning properties to strain values and finds a linear relationship of approximately 3:1 between Von Mises strain and material thinning percentage throughout the sample. The slope of the line appears to correlate strongly with the material's Poisson's ratio and could have potential uses in process planning.
\end{abstract}

\section{INTRODUCTION}

Hydroforming is a near net shape manufacturing process that is currently used in many different industries to create complicated geometries from readily available sheet and tube metal blanks. Hydroforming works in a similar fashion to traditional mechanical pressing except using fluid pressure instead of (or in conjunction with) other mechanical forces. One of the main concerns with any forming process is the condition of the material when the forming operation is complete. Industrial applications typically require hydroformed components to meet geometric specification that range from external dimensions to minimum wall thicknesses and maximum strain values. Given a component specified in these terms, a manufacturing engineer must determine the blank geometry that will deliver the required part after forming. Motivated by the need to provide manufacturing engineers with a simple quantitative relationship between local wall thickness and local surface strain the authors investigated the values of strain developed during the hydroforming of a hemisphere.

One of the ways to measure the post-forming strain condition on the surface of the material is to etch a sample with a circular pattern before a forming operation and analyze the resulting pattern with a surface strain analysis using a CGA technique. This technique examines the surface pattern and determines how it has changed, moved or otherwise distorted which allows for the computation of strain. There are many ways to mark and examine samples but this analysis used a $2 \mathrm{~mm}$ diameter "single circle grid" using a position based system because it has been shown 
to be accurate in similar shapes by previous work such as Ozturk, et al., 2009. The type of geometry used is similar to ones found in the literature such as Spence, et al., who looked at strain measurements in a domed sample and found that the highest levels of strain were in the transition area between the domed section and the flange. The specific technique chosen for this analysis used multiple sets of high resolution photographs and the GOM ARGUS system developed by GOM to analyze them.

A common method used to accurately measure the geometry of a sample is through optical measurement techniques such as the GOM ATOS system which is also developed by GOM. This technique uses high resolution cameras and blue light technology to scan the surface of a part and create a point cloud which precisely defines the surface of a sample, generates an accurate geometric representation, and creates information which is directly compatible with the ARGUS system. Both these types of analyses are performed on the sample in this study and the results are compared.

\section{Hydroforming Process}

The equipment used for forming the sample was a Triform sheet hydroforming press (model 610-20-3SC) equipped with deep draw capability and a bladder separating the workpiece from the acting fluid. Before the forming operation, the blank was cut into a circular section, lubricated and placed in the hydroforming machine on top of the draw ring and punch. Then fluid was pumped into the chamber until the chamber was full, and then the pressure was increased substantially while the punch simultaneously traveled upwards. Because of the large amount of fluid pressure, the blank was forced tightly against the punch and conformed precisely to its shape. The goal of the pressure cycle is to create fluid pressure high enough to prevent wrinkling and low enough to allow material to draw into the working zone (which helps avoid tearing).

\section{Material}

The material used was a $1 \mathrm{~mm}$ thick sheet of Ti35A known as "commercially pure" titanium. The blank was cut with a water jet to a diameter of $127 \mathrm{~mm}$.

Maximum chemical composition (\% wt.)

\begin{tabular}{|c|c|c|c|c|c|c|c|}
\hline $\mathrm{O}$ & $\mathrm{N}$ & $\mathrm{C}$ & $\mathrm{Fe}$ & $\mathrm{H}$ & $\begin{array}{c}\text { Residual } \\
\text { each }\end{array}$ & $\begin{array}{c}\text { Residual } \\
\text { total }\end{array}$ & $\mathrm{Ti}$ \\
\hline 0.18 & 0.03 & 0.08 & 0.20 & 0.015 & 0.10 & 0.40 & Else \\
\hline
\end{tabular}

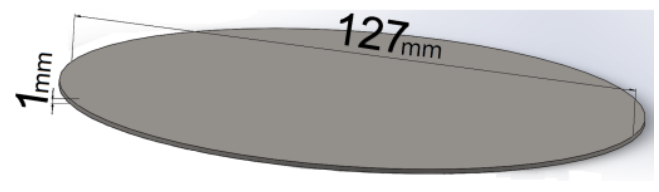

FIGURE 1. Chemical Composition (Timet, 2016)

FIGURE 2. Blank Size

\section{Surface Strain Analysis (GOM ARGUS)}

The final shape of the sample is shown in Fig. 3 it is a small domed geometry with a tear a little less than half way around the circumference. In order to properly assess all of the points on the surface, the sample was mounted to a rotating table and 16 pictures were taken around the circumference of the sample at $20^{\circ}, 45^{\circ}$, and $75^{\circ}$ angles with an additional 8 photos taken from almost directly above. This yielded a total of 56 pictures for the analysis which were used to stitch together a point cloud that defined the surface of the object. The sample geometry can be seen below in Fig. 3A with the picture locations in Fig. 3B and the point cloud detection in Fig. 3C.

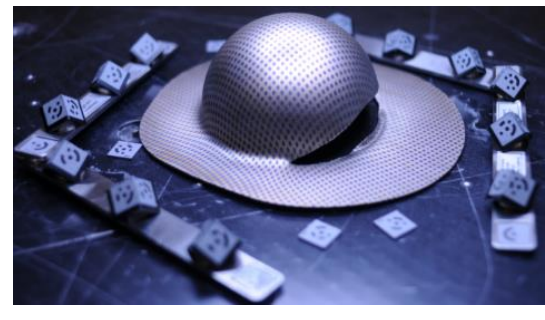

(a) Sample Setup

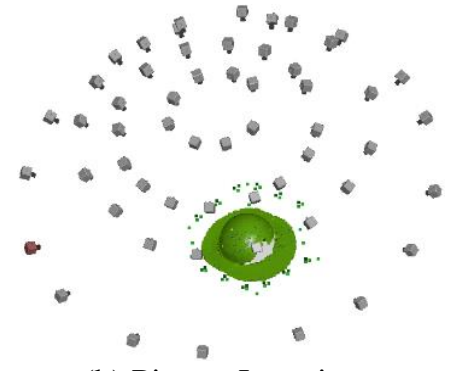

(b) Picture Locations

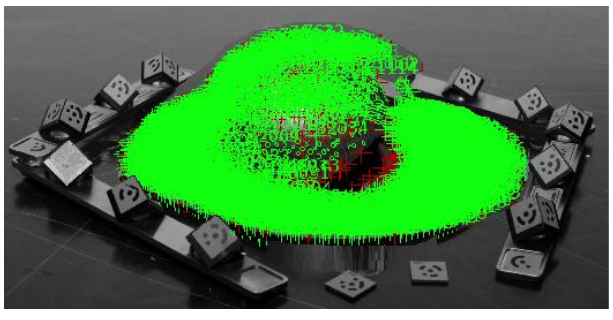

(c) Point Cloud Detection

FIGURE 3. Setup for Strain Analysis 
The point cloud is a database of positional values. By using the $2 \mathrm{~mm}$ diameter etched dots on the surface, values for both the Von Mises strain and the material thinning percentage are calculated and matched with the position. The highest levels of Von Mises strain were observed on the radius of the transition area of the sample where the flange leads into the bulge. The highest values are in the range of $70-80 \%$ and were located on the opposite side of the failure. In this case it is most likely that this area was most stressed around this transition area and the failure on one side inhibited high strain concentrations in its vicinity. Similarly, the areas with highest material thinning (25-30\%) can also be seen in this area. It is also worth noting that because of the tear it is impossible to get strain measurements right next to the failure zone. The Von Mises strain percentage map obtained from the ARGUS system can be seen in Fig. 4. The map of major versus minor strain gathered from the surface can be seen below in Fig. 5.

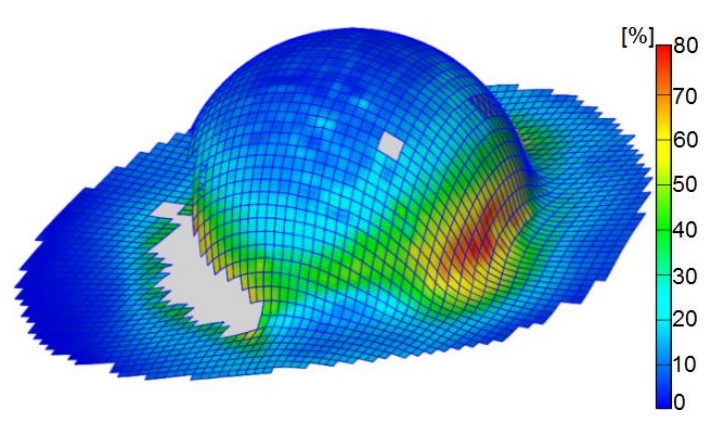

FIGURE 4. Von Mises Strain Percentage

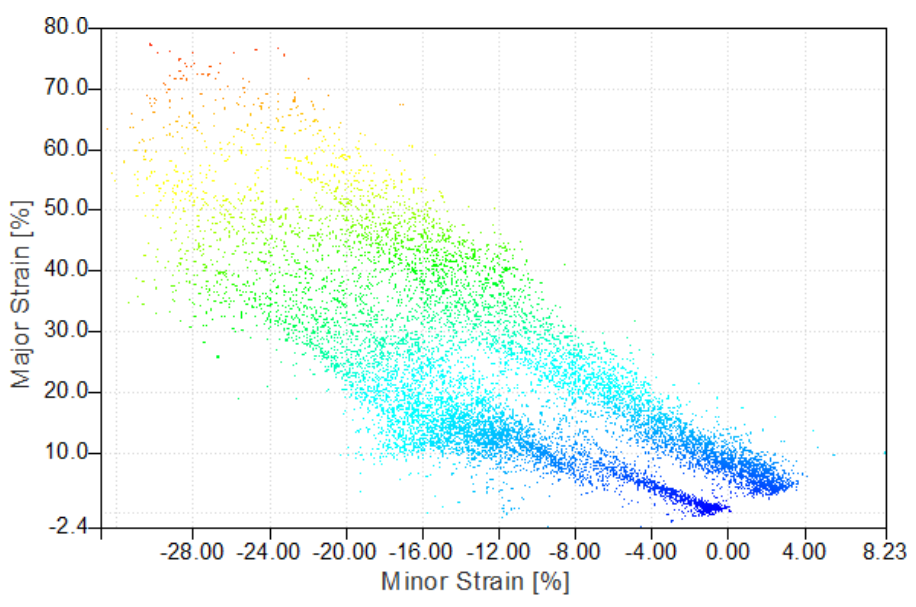

FIGURE 5. Major vs Minor Strain

\section{Geometric Analysis (GOM ATOS)}

The sample was scanned on all sides by an ATOS optical measurement system to achieve an accurate profile of geometric information. This information was gathered into a point cloud separate from the one generated by the ARGUS system described previously. Since the ATOS system is purpose built to accurately study geometry and can scan an entire part at once instead of one side, the geometrical information (including material thinning percentage) is more accurate than that from the ARGUS system.
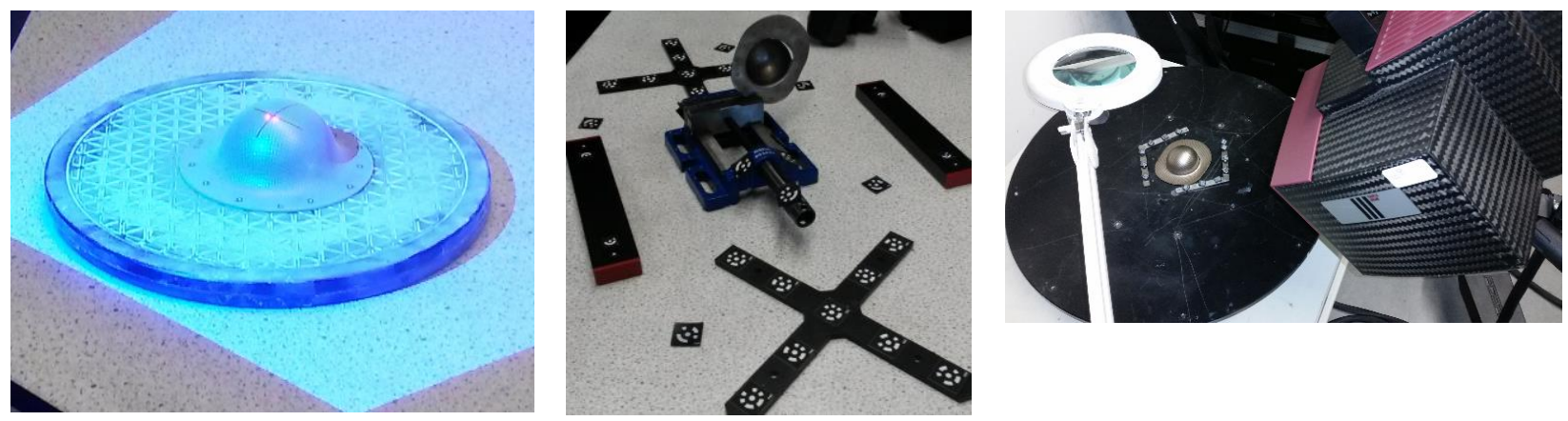

FIGURE 6. GOM Part Scanning and Setup

\section{Data Preparation}

After individual data sets were collected from both the ATOS and ARGUS systems, the data was paired and examined for anomalies. To ensure the pairing was accurate, the distance between the points where thickness and strain were measured needed to be in close proximity. For this reason, points which were 3 standard deviations 
outside the average were removed. Because the average and standard deviation were $0.19 \mathrm{~mm}$ and $0.24 \mathrm{~mm}$ respectively, this ensured that the paired strain and thinning measurements were within $1 \mathrm{~mm}$ of each other. Examining the relationship between the thickness reduction and strain values yields a positive correlation between the wall thickness and the Von Mises strain as can be observed in Fig. 7.

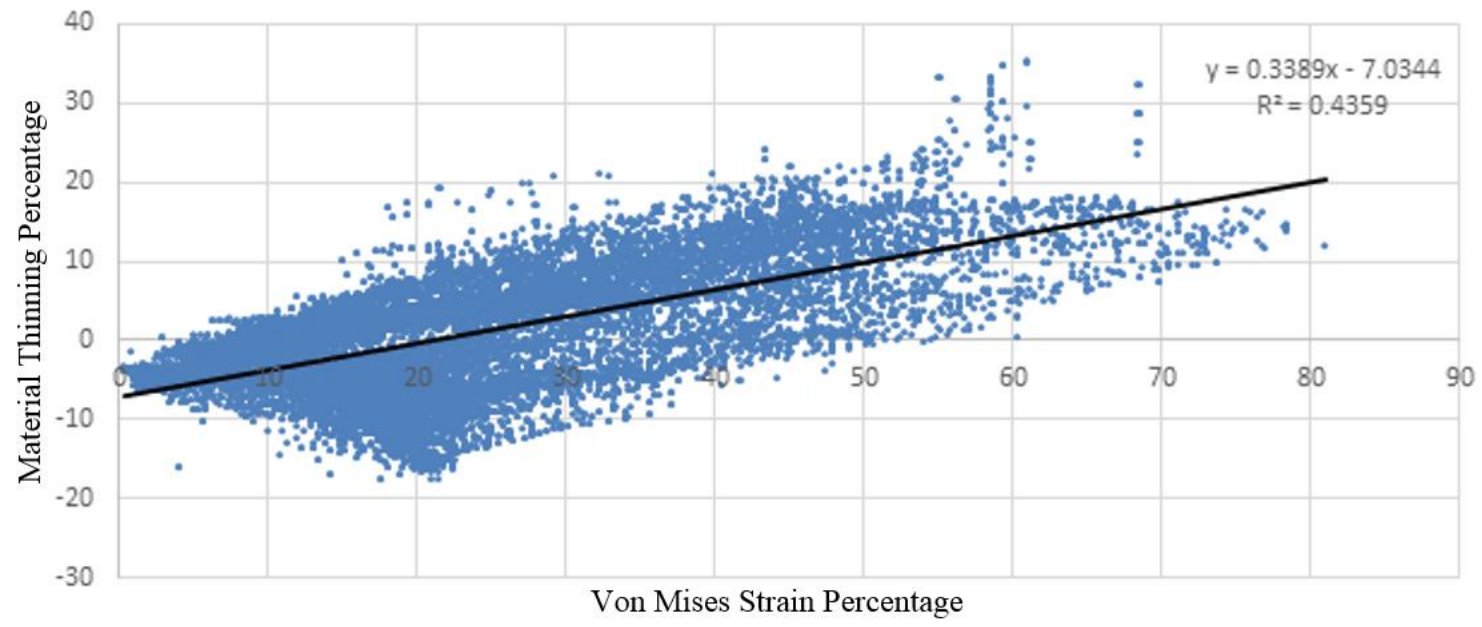

FIGURE 7. Function of Ti35A Von Mises Strain and Material Thinning Obtained by Linear Regression

\section{Defining Specific Sections of Interest}

In order to isolate the points on the top of the dome a subsection of data was chosen for further analysis. In this case two sections across the length of the sample were defined, one traversed the cracked area, the other ran orthogonally and did not. The coordinate system was defined in such a way that the red line in Fig. 8 runs in the $\mathrm{X}$ direction, and the green line in the $\mathrm{Y}$ (with the $\mathrm{Z}$ direction upwards). A $2 \mathrm{~mm}$ width was defined and the points that fell within were included into the data subsets for an independent linear regression analysis. The width of the band was defined to be $2 \mathrm{~mm}$ wide with the intent of being small enough to ensure the data on top of the dome could be properly examined and was not overwhelmed by other points (like it is in Fig. 7), while still being large enough to produce an adequate amount of data for the analysis.

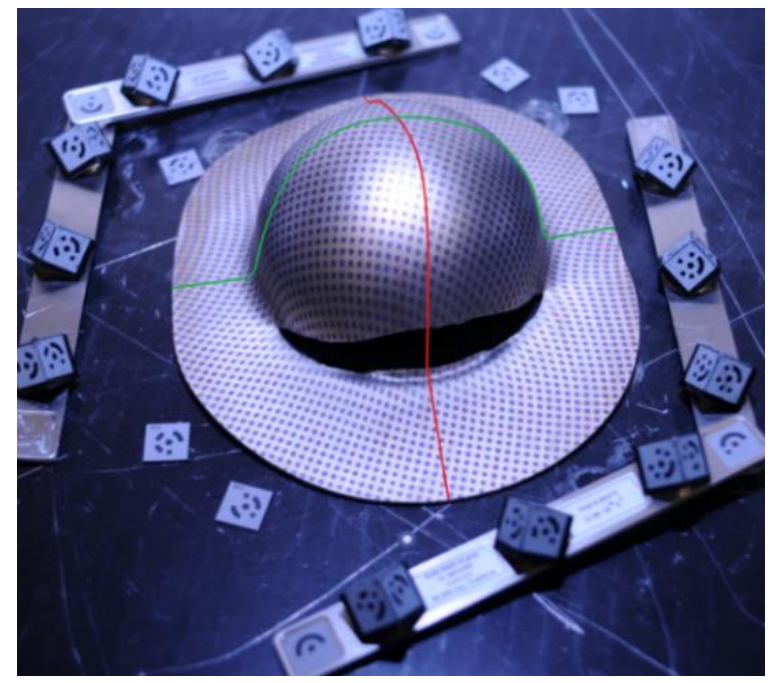

FIGURE 8. Definition of Cross Sections

The section running across the crack in the "X direction" showed a similar correlation between material thinning percentage and strain as observed in Fig. 7 except with a higher degree of correlation and a 3:1 instead of 2.5:1 ratio 
between strain and thinning. In contrast, the result for the data set in the "Y Direction" that did not traverse the crack did not show a definitive correlation. These results can be seen below in Fig. 9 and Fig. 10.

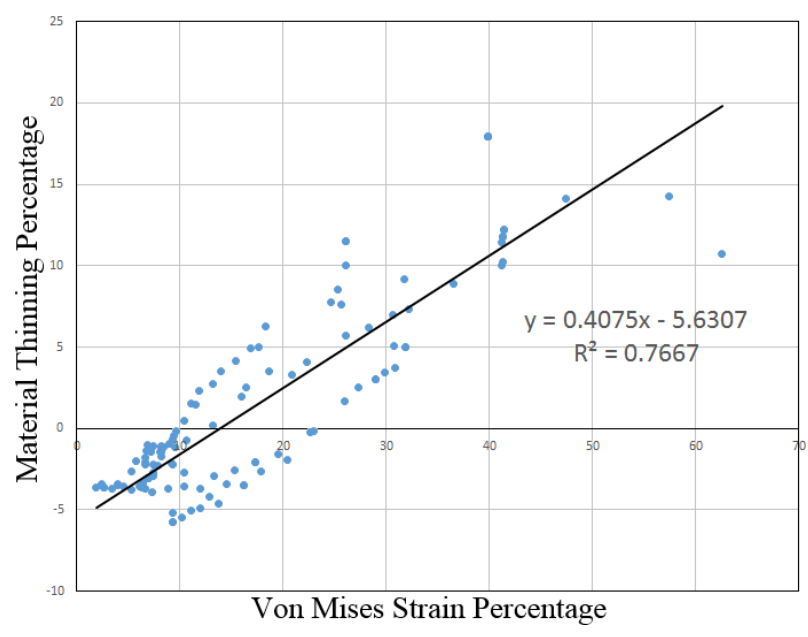

FIGURE 9. Strain Versus Thinning (X Direction)

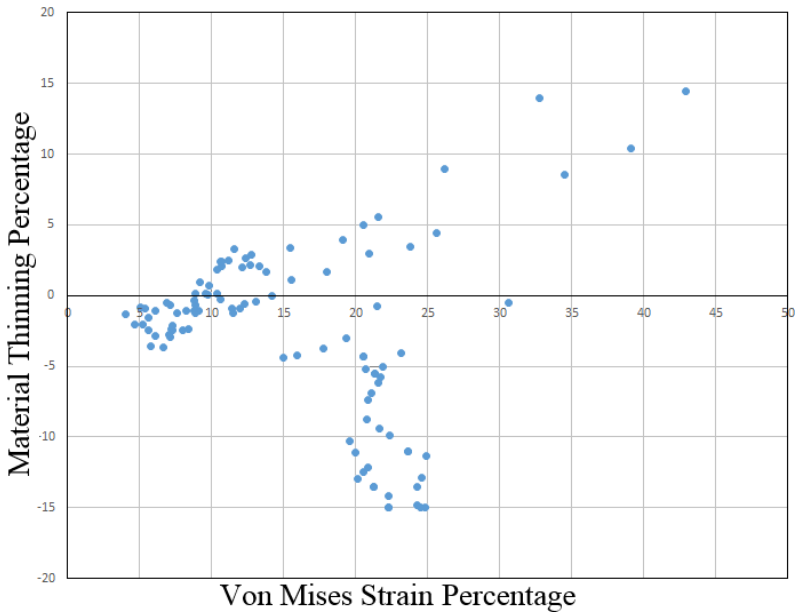

FIGURE 10. Strain Versus Thinning (Y Direction)

\section{Discussion}

The post processing analysis shows a linear correlation between the Von Mises strain percentage and the material thinning percentage of 3:1, which correlates closely with the materials Poisson's ratio of 0.3. When looking at the specific area of interest which crosses the crack this relationship is similar (at around 2.5:1) but with a significantly higher $\mathrm{R}$ value of 0.77 instead of 0.44 (an $\mathrm{R}$ value is a calculated measure of how well an equation fits a data set where 1 is a perfect fit). However, the area of interest which does not traverse the crack did not seem to share this correlation. This is because there are a large number of points which show a significant material thickening (negative thickness reduction) clustered at a strain rate of around 20-25\% which seemed to be an anomaly caused by the crack interfering with the data set that crossed it. Upon a close inspection of all points that had significant material thickening (i.e. the aforementioned region of anomaly), it was discovered that all significant thickening in the data set occurred at the edge of the flange.

The most plausible explanation is that as the hydroforming operation occurs, the material in the flange thickens as it is pulled inwards and forced to adopt a smaller radius. However this material thickening does not occur in the data set that crossed the crack and the reason for this is that further material draw would have been inhibited in this direction after the onset of the crack. In other words, further deformation in this area would have simply enlarged the tear which would have in turn inhibited further thickening in the flange as it would not have been forced to adopt a smaller radius. If the data cluster in the flange was to be discounted as the edge of the flange area may not be relevant during an industrial process, the same general trend is found with this data set.

\section{Limitations}

A limitation of the analysis can be observed on the top surface in the ARGUS results of the domed area of the sample. In this region the software that calculates the surface points, only identified a proportion of the points automatically meaning that others had to be identified and input into the point cloud manually. The points which were input manually were not found automatically because of the difficulty the software had in identifying the points on the surface of the sample due to the contrast issues caused by shadows in the photographs. While various sets of pictures were taken with different light sources and filters, even the final set of images used for the analysis contained points on the surface and around the radius which needed manual identification which is visible on the top of the domed sample as a slightly wavy texture instead of a smooth surface. While this adds variation, the values at these points are close to the adjacent points and this indicates that the inaccuracies are probably relatively minor. Examples of these inaccurate areas are shown in Fig. 11. 


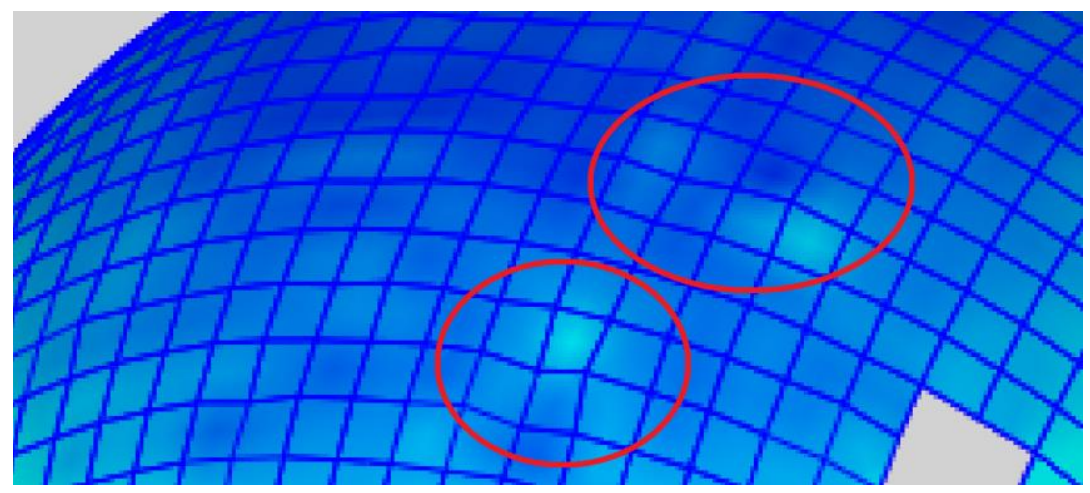

FIGURE 11. Inaccuracies in the Mesh

\section{Further Work}

This work could be expanded upon by comparing various punch heights and seeing when failure occurs (either experimentally or with simulations). It could also be suggested to test different grades and thicknesses of material to observe whether relationships occur in the same or different proportions, i.e. whether the 3:1 strain to thinning relationship is also observed in different materials and sizes. Finally, one useful addition to this work would consist in analyzing the sample with microscopy to observe how the texture of the metal varies in different locations, specifically around the failure and in the areas of higher strain concentration and thinning.

\section{CONCLUSION}

This paper discusses the results from a CGA analysis of a titanium alloy sample which was formed beyond the point of failure during a hydroforming process. The results yielded the highest concentrations of Von Mises strain and the most pronounced material thinning in the transition zone on the opposite side of the failure. Two subsets of data which included points running across the top of the sample were defined and the correlation between strain and material thinning was examined which showed a correlation of approximately 3:1 between Von Mises strain and material thinning percentage. This result is similar to conclusions described in the literature (Spence, et al., 2008) as the highest levels of strain are also observed in the transitional area between the flange and the domed section.

\section{ACKNOWLEDGMENTS}

The authors would like to acknowledge and thank Triform presses (a division of Beckwood presses) for forming the sample used in this research and allowing us to publish the results. The authors would also like to thank the core members of the Advanced Forming Research Centre for their support, providing information and material, and for allowing this work to be published.

\section{REFERENCES}

1. Keeler, S., 2003. Training Manual: Enhanced FLC Project, The Auto/Steel Partnership, Southfield, MI. [Online]

Available at: http://www.a-sp.org/ /media/Files/ASP/Lightweight\%20Programs/Technology\%20ReportFinal.pdf

[Accessed 2012 2016].

2. Ozturk, F. et al., 2009. Grid Marking and Measurement Methods for Sheet Metal Formability. s.1., s.n., pp. 18-21.

3. Spence, A. D. et al., 2008. Simultaneous Large Scale Sheet Metal Geometry and Strain Measurement. Journal of Manufacturing Science and Engineering, 130(5).

4. Timet, 2016. Ti35A Datasheet. [Online] Available at: http://www.timet.com/datasheets-and-literature [Accessed 1912 2016]. 